\title{
A convenient method for the reductive degradation of mono-, di-, and tribromodiphenyl ethers, tetrabromo- and tetrachlorobisphenol A with Raney Ni-Al alloy
}

\author{
Guo-Bin Liu, *a Hong-Yun Zhao, ${ }^{a}$ Lu Dai, ${ }^{\text {a }}$ and Thies Thiemann ${ }^{\text {b,c }}$ \\ ${ }^{a}$ Department of Chemistry, Fudan University, 220 Handan Road, Shanghai 200433, P. R. China. \\ ${ }^{b}$ Interdisciplinary Graduate School of Engineering Sciences, Kyushu University, 6-1, \\ Kasuga-koh-en, Kasuga, Fukuoka 816-8580, Japan \\ ${ }^{c}$ new address: Department of Chemistry, United Arab Emirates University, PO Box 17551, \\ Al Ain, UAE \\ E-mail: liuguobin@fudan.edu.cn
}

\begin{abstract}
Raney Ni-Al alloy in a dilute aqueous alkaline solution of $\mathrm{NaOH}, \mathrm{KOH}, \mathrm{CsOH}, \mathrm{LiOH}$, or $\mathrm{Ca}(\mathrm{OH})_{2}$, or of alkali metal carbonates such as $\mathrm{Na}_{2} \mathrm{CO}_{3}, \mathrm{~K}_{2} \mathrm{CO}_{3}$ and $\mathrm{Cs}_{2} \mathrm{CO}_{3}$, becomes a very powerful reducing agent and dehalogenates mono-, di and tribromodiphenyl ethers (BDPEs), tetrabromobisphenol A (TBBPA) and tetrachlorobisphenol A (TCBPA) effectively. Polybromodiphenyl ethers are reduced to give diphenyl ether as sole product. TBPPA and TCPPA are debrominated to bisphenol A and other dehalogenated compounds. The C-C bond of bisphenol $\mathrm{A}$ is broken easily under the reaction conditions. An almost quantitative removal of halogen from BDPEs, TBBPA and TCPPA can be realized in aqueous media under mild reaction conditions without the use of organic solvents. The reductive dehalogenation of TBBPA and TCBPA with a Raney Ni-Al alloy under ultrasonication is also described.
\end{abstract}

Keywords: Dehalogenation, polyhalogenated aromatics, Raney Ni-Al alloy, remediation

\section{Introduction}

Recently, a great deal of attention has been paid to environmental pollution caused by chlorinated and brominated organic aromatic compounds such as polybromodiphenyl ethers (PBDPEs), tetrabromobisphenol A (TBBPA) and tetrachlorobisphenol A (TCBPA) as these are hazardous and long-lived once released into the biosphere. Over many years, TBBPA, TCBPA and PBDPEs have been utilized as flame retardants in the plastics industry. ${ }^{1}$ The use of brominated and chlorinated flame retardants is still on the increase and is strongly linked to their presence in appliances for use in homes and offices such as TVs, computers, and other pieces of 
electronic equipment. Flames retardants are also utilized in fabrics, paints, coatings and furnishings for car interiors. Thus, PBDPEs, TBBPA and TCBPA have become ubiquitous in our environment, having been detected not only in sewage sludge, in household air, and in dust, but also as contaminants in the eggs of birds, including chickens, in farmed and wild salmon, ${ }^{2}$ and in human serum. ${ }^{3}$ While the in vivo properties of PBDEs, TCBPA and TBBPA are hard to quantify, ${ }^{2 a, 4}$ they have been shown to be biologically active, in both in vitro and in vivo studies. $^{2 \mathrm{a}, 4}$ The impact of PBDPEs, TBBPA and TCBPA on the environment is not clear, but their potential toxicity has led to some calls for an urgent substitution of these and related halogenated compounds in commercial products. ${ }^{5}$ Obviously, even a rapid and comprehensive substitution of PBDPEs, TBBPA and TCBPA results in the serious problem of waste elimination of existing materials in which PBDPEs, TBBPA and TCBPA have been widely utilized. A viable elimination of PBDPEs, TBBPA and TCBPA wastes in spent products, in sludge and in other matrices presents a very serious problem and efforts are being undertaken to find new methods of remediation. It has been shown that direct thermal decomposition of TBBPA at temperatures above $210{ }^{\circ} \mathrm{C}$ leads to a host of brominated phenols and brominated bisphenols. ${ }^{6}$ It is known that upon incineration of municipal waste, PBDPEs, TBBPA and TCBPA can also give rise to highly toxic polybrominated or polychlorinated dibenzo- $p$-dioxins and dibenzofurans. ${ }^{7}$ The addition of polypropylene and polyethylene to TBBPA in a pyrolysis experiment in an encapsulated ampoule under an inert atmosphere has been shown to reduce significantly the amount of brominated phenols formed. ${ }^{8}$ Debromination of tetrabromobisphenol A in epoxy resins has been achieved with $\mathrm{Na} / \mathrm{NH}_{3}$ in an autoclave at $100{ }^{\circ} \mathrm{C}$ and $6 \mathrm{MPa}^{9}$ and has also been carried out with ethyl acetate at $280{ }^{\circ} \mathrm{C}$ and $15 \mathrm{MPa} .{ }^{10}$ At lower temperatures and at normal pressure, partial dehalonitration may be mentioned, in which TBBPA is converted with nitric acid in an organic solvent to a dinitro-dibromobisphenol. ${ }^{11}$ Moreover, reductive debromination with iron $(0)$ and photodegradation of PBDPEs were reported, ${ }^{12}$ although the debrominating efficiency is poor, though better results have been obtained by photodegradation in the presence of $\mathrm{TiO}_{2} \cdot{ }^{13}$ Recently, M. Brebu and Y. Sakata studied a novel debromination method for flame retardants containing high impact polystyrene, by ammonia treatment. ${ }^{14}$

Against this background, the authors have searched for other methods of dehalogenating polybromo- and polychlorinated aromates and have turned to Raney Ni-Al alloy. Raney Ni has been extensively utilized as a catalyst for the hydrodechlorination of persistent organic pollutants (POPs) such as dieldrin, DDT and lindane under a hydrogen atmosphere. ${ }^{15}$ However, Raney NiAl alloy itself, from which Raney Ni catalyst is derived, has attracted limited attention for this type of hydrodechlorination. In the course of our research aiming at developing new ways of degrading hazardous wastes, ${ }^{16}$ we have assessed the utilization of Raney Ni-Al alloy directly, using the active Raney Ni metal formed in situ as a catalyst for the dehydrohalogenation of halogenated aromatic POPs. 


\section{Results and Discussion}

Raney Ni-Al alloy, which is commercially available, cheap and easy to handle, has been widely employed as a starting material for preparing Raney Ni catalyst and as a reductant in 10-25\% $\mathrm{NaOH}$ aqueous solution in organic synthesis. ${ }^{15}$ In a preliminary communication, we reported that Raney Ni-Al becomes a very strong reducing agent in dilute aqueous alkaline solutions and that it reduces tetrabromobisphenol A easily to give bisphenol A and C-C cleavage products such as phenols and cyclohexanols as their hydrogenated derivatives. ${ }^{16 \mathrm{~b}}$ The method is carried out under mild conditions and in the absence of organic solvents. ${ }^{16}$ An obvious advantage of this method is that water is employed both as solvent and proton source. The reduction is carried at atmospheric pressure. No special apparatus is required. These successful results prompted us to develop a convenient and practical method for the disposal of halogenated flame retardants in general. In this paper, we give a detailed report on the reductive dehalogenation of tetrabromo- and tetrachlorobisphenol A, and of mono-, di- and tribromodiphenyl ethers with Raney Ni-Al alloy in dilute basic aqueous solutions. The results are shown in Schemes 1-3 and Tables 1-8.

\section{Dehalogenation of tetrabromo- and tetrachlorobiphenol A (1a,b)}

Effects of the nature of the aqueous alkaline solution, the amount of the Raney Ni-Al alloy used and the reaction temperature
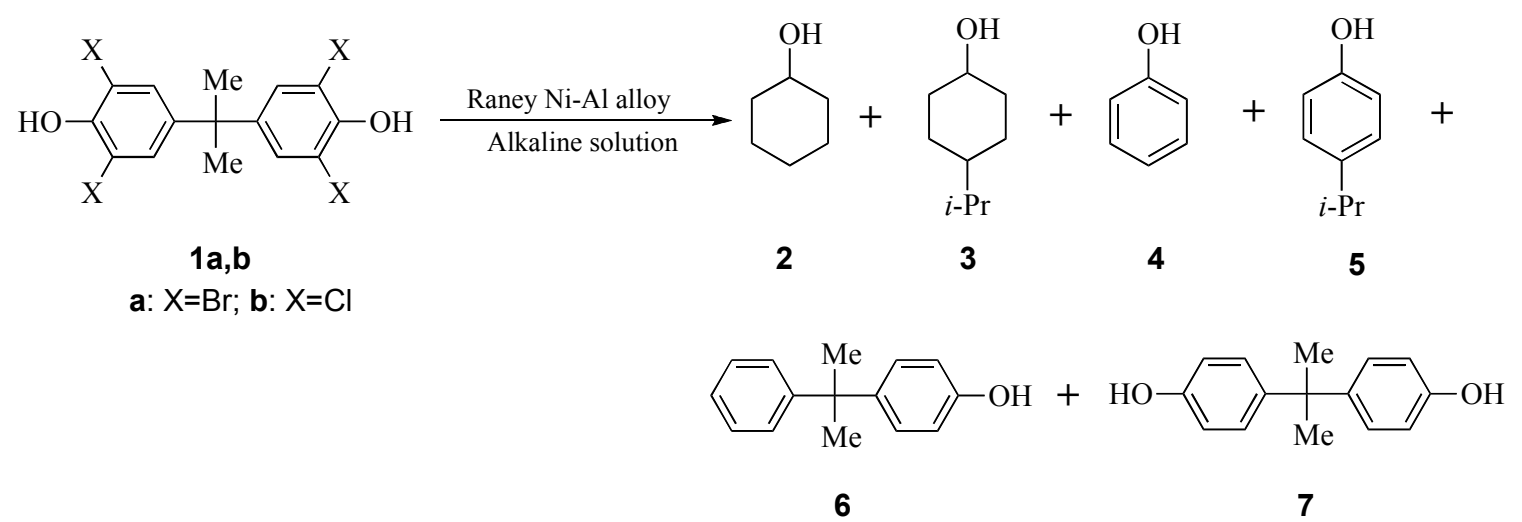

Scheme 1. Poducts found in the reaction of bisphenols 1a,b with Raney Ni-Al alloy

As shown in Scheme 1 and Table 1, tetrabromobisphenol A (TBBPA, 1a) was debrominated easily by using Raney Ni-Al alloy in a dilute aqueous alkaline solution. The debromination was carried out by slowly adding an aqueous alkaline solution to a mixture of tetrabromobisphenol A 1a, Raney Ni-Al alloy and water and then heating the resulting mixture at $90{ }^{\circ} \mathrm{C}$. The debromination was monitored by GC and GC-MS. Initially, as inorganic bases, $\mathrm{KOH}, \mathrm{CsOH}$ and $\mathrm{NaOH}$ were employed, all at $1 \mathrm{wt} \%$. 1a was reduced to give bisphenol A 7 as the major product, together with small amounts of cyclohexanol 2, 4-isopropylcyclohexanol 3, phenol 4, 4-isopropylphenol 5 and 2hydroxyphenyl-2-phenylpropane 6 (Runs 1-4). In the case of adding a $1 \mathrm{wt} \%$ aq. $\mathrm{NaOH}$ solution, 
the GC ratio of compound $7(55.0 \% \rightarrow 77.5 \%)$ increased (Table 1 , Run 2 vs Run 3), when the quantity of Raney Ni-Al alloy was decreased $(5 \mathrm{~g} \rightarrow 2.5 \mathrm{~g})$. Other alkali and alkali earth hydroxides such as $\mathrm{Ca}(\mathrm{OH})_{2}, \mathrm{LiOH}$, and $\mathrm{Ba}(\mathrm{OH})_{2}$, when employed as $1 \mathrm{wt} \%$ aq. solutions, gave similar mixtures of reduction products $\mathbf{2}, \mathbf{3}, \mathbf{4}, \mathbf{5}, \mathbf{6}$ and 7 (Runs 5-8, Table 1).

Table 1. Effects of the concentration of alkaline solution, the amount of Raney Ni-Al alloy used and the reaction temperature in hydrodebromination of tetrabromobisphenol $\mathbf{1} \mathbf{a}^{\mathrm{a}}$

\begin{tabular}{|c|c|c|c|c|c|c|c|c|c|c|}
\hline \multicolumn{6}{|c|}{ Ratio $(\%)^{\mathrm{c}, \mathrm{d}}$} & \multirow[t]{2}{*}{ (h) } & \multirow{2}{*}{$\begin{array}{l}\text { Temp } \\
\left({ }^{\circ} \mathrm{C}\right)\end{array}$} & \multirow{2}{*}{$\begin{array}{l}\text { Alkaline solution }^{\mathrm{b}} \\
\qquad(\mathrm{mL})\end{array}$} & \multirow{2}{*}{$\begin{array}{l}\mathrm{Ni} / \\
\mathrm{Al} \\
(\mathrm{g})\end{array}$} & \multirow[t]{2}{*}{ Run } \\
\hline 7 & 6 & 5 & 4 & 3 & 2 & & & & & \\
\hline $\begin{array}{c}48.9 \\
(44.2)\end{array}$ & $\begin{array}{c}6.3 \\
(4.1)\end{array}$ & $\begin{array}{c}27.2 \\
(22.7)\end{array}$ & 0 & $\begin{array}{c}9.3 \\
(6.2)\end{array}$ & $\begin{array}{c}8.3 \\
(5.4)\end{array}$ & 5 & 90 & $1 \% \mathrm{KOH}(100) / \mathrm{H}_{2} \mathrm{O}(100)$ & 5 & 1 \\
\hline 55.0 & 6.0 & 23.4 & 0 & 8.1 & 7.5 & 6 & 90 & $1 \% \mathrm{NaOH}(100) / \mathrm{H}_{2} \mathrm{O}(100)$ & 5 & 2 \\
\hline 77.5 & 2.2 & 14.4 & 0 & 3.1 & 2.8 & 8 & 90 & $1 \% \mathrm{NaOH}(100) / \mathrm{H}_{2} \mathrm{O}(100)$ & 2.5 & 3 \\
\hline $\begin{array}{c}49.7 \\
(44.9)\end{array}$ & $\begin{array}{c}6.6 \\
(4.7)\end{array}$ & $\begin{array}{c}24.1 \\
(20.6)\end{array}$ & 0 & $\begin{array}{l}10.3 \\
(7.1)\end{array}$ & $\begin{array}{c}9.3 \\
(5.9)\end{array}$ & 5 & 90 & $1 \% \mathrm{CsOH}(100) / \mathrm{H}_{2} \mathrm{O}(100)$ & 5 & 4 \\
\hline 57.4 & 6.1 & 22.4 & 0 & 8.4 & 5.7 & 5 & 90 & $\begin{array}{c}1 \% \mathrm{Ca}(\mathrm{OH})_{2}(100) \\
/ \mathrm{H}_{2} \mathrm{O}(100)\end{array}$ & 5 & 5 \\
\hline 62.0 & 5.6 & 20.6 & 0 & 7.8 & 4.0 & 6 & 90 & $1 \% \mathrm{LiOH}(100) / \mathrm{H}_{2} \mathrm{O}(100)$ & 5 & 6 \\
\hline 66.7 & 4.8 & 18.0 & 0 & 7.1 & 3.4 & 6 & 90 & $\begin{array}{c}1 \% \mathrm{Ba}(\mathrm{OH})_{2}(100) \\
/ \mathrm{H}_{2} \mathrm{O}(100)\end{array}$ & 5 & 7 \\
\hline 89.0 & 7.9 & 1.9 & 0 & 0.6 & 0.6 & 4 & 90 & $0.2 \% \mathrm{KOH}(100) / \mathrm{H}_{2} \mathrm{O}(100)$ & 2 & 8 \\
\hline 90.7 & 6.9 & 1.6 & 0 & 0.4 & 0.4 & 4 & 90 & $\begin{array}{c}0.2 \% \mathrm{NaOH}(100) \\
/ \mathrm{H}_{2} \mathrm{O}(100)\end{array}$ & 2 & 9 \\
\hline 88.0 & 8.5 & 2.3 & 0 & 0.8 & 0.8 & 5 & 90 & $0.2 \% \mathrm{CsOH}(100) / \mathrm{H}_{2} \mathrm{O}(100)$ & 2 & 10 \\
\hline 91.1 & 4.7 & 2.3 & 0.6 & 0.8 & 0.8 & 5 & 90 & $\begin{array}{c}0.2 \% \mathrm{Ca}(\mathrm{OH})_{2}(100) \\
/ \mathrm{H}_{2} \mathrm{O}(100)\end{array}$ & 2 & 11 \\
\hline 92.6 & 5.5 & 1.0 & 0.5 & 0.1 & 0.1 & 5 & 90 & $0.2 \% \mathrm{LiOH}(100) / \mathrm{H}_{2} \mathrm{O}(100)$ & 2 & 12 \\
\hline 94.7 & 3.7 & 0.9 & 0.4 & 0.1 & 0.1 & 5 & 90 & $\begin{array}{c}0.2 \% \mathrm{Ba}(\mathrm{OH})_{2}(100) \\
/ \mathrm{H}_{2} \mathrm{O}(100)\end{array}$ & 2 & 13 \\
\hline 84.9 & 2.9 & 7.2 & 1.3 & 1.9 & 1.9 & 9 & 60 & $1 \% \mathrm{NaOH}(100) / \mathrm{H}_{2} \mathrm{O}(100)$ & 5 & 14 \\
\hline 77.3 & 3.7 & 10.8 & 2.5 & 2.2 & 2.2 & 6 & $60^{\mathrm{e}}$ & $1 \% \mathrm{NaOH}(100) / \mathrm{H}_{2} \mathrm{O}(100)$ & 5 & 15 \\
\hline 98.4 & 0 & 0.8 & 0.8 & 0 & 0 & 5 & $60^{\mathrm{e}}$ & $\begin{array}{c}0.2 \% \mathrm{NaOH}(100) \\
/ \mathrm{H}_{2} \mathrm{O}(100)\end{array}$ & 2 & 16 \\
\hline
\end{tabular}

${ }^{\mathrm{a}} 1 \mathrm{a}(2.0 \mathrm{mmol}, 1.09 \mathrm{~g})$. ${ }^{\mathrm{b}}$ added within $1.0 \mathrm{~h} .{ }^{\mathrm{c}} \mathrm{GC}$ ratio. ${ }^{\mathrm{d}}$ isolated yields in parentheses. e ultrasonication.

Compound 4 could be formed via reductive C-C bond cleavage in $\mathbf{1 a} / \mathbf{b}, \mathbf{7}$, in a partially debrominated intermediate or from a combination of these. Compounds $\mathbf{2}$ and $\mathbf{3}$ are produced by 
hydrogenation of phenols $\mathbf{4}$ and $\mathbf{5}$ under the reaction conditions. While bond cleavage in BPA is known to occur under a number of conditions, such as by enzymatic reaction, ${ }^{17}$ thermolysis at above $150{ }^{\circ} \mathrm{C}^{18}$ and photodegradation, ${ }^{19}$ it is unusual that this cleavage can also take place under reductive conditions at relatively low temperatures, although cleavage reactions of bisphenols are known to be base-catalysed. ${ }^{20}$ The occurrence of $\mathbf{6}$, as deoxygenated $\mathbf{7}$, is more interesting. Generally, the deoxygenation of non-activated phenols is not easy ${ }^{21}$ and mostly occurs under conditions found in hydroprocessing. ${ }^{22}$ Bisphenol-A 7 was afforded in much higher yield (Runs 8 and 9 vs runs 1 and 2, Table 1) in a $0.2 \mathrm{wt} \% \mathrm{KOH}$ solution. Thus, results indicate that the concentration of the base affects the ratio of the debromination products greatly. In more dilute alkaline conditions, products stemming solely from dehalogenation are formed overwhelmingly, while C-C cleavage products of the bisphenols, such as phenols $\mathbf{4}$ and $\mathbf{5}$, and cyclohexanols $\mathbf{2}$ and 3 were found only in trace amounts.

The debromination of $\mathbf{1 a}$ at lower temperature $\left(60^{\circ} \mathrm{C}\right)$ under ultrasonication was examined. A mixture of compounds $2, \mathbf{3}, \mathbf{4}, \mathbf{5}$ and $\mathbf{6}$ was obtained, when carrying out the reaction by dropwise addition of an aq. $1 \mathrm{wt} \% \mathrm{NaOH}$ solution (Table 1, Run 15). Ultrasonic irradiation enhanced the reaction markedly. Without ultrasonic irradiation, the starting material was consumed after $9 \mathrm{~h}$ (Table 1, Run 14). The same reaction under ultrasonication, under otherwise identical conditions, was complete after $6 \mathrm{~h}$.

The silent reaction furnished almost exclusively pure debrominated product, while ultrasonication led to some $\mathrm{C}$ - $\mathrm{C}$ cleavage. In more dilute basic solutions, the reaction became markedly slower at $60{ }^{\circ} \mathrm{C}$ (vs. $90{ }^{\circ} \mathrm{C}$ ), and the difference between the silent reaction and the reaction under ultrasonication became more pronounced. In the case of adding an aq. $0.2 \mathrm{w} \%$ $\mathrm{NaOH}$ solution at $60{ }^{\circ} \mathrm{C}$ and under ultrasonication, compound $\mathbf{6}$ was formed as the main product, along with trace amounts of phenols $\mathbf{4}$ and $\mathbf{5}$. No cyclohexanols $\mathbf{2}$ and $\mathbf{3}$ were detected. However, without ultrasonication, the debromination became sluggish at $60{ }^{\circ} \mathrm{C}$, and $46 \%$ of $1 \mathrm{a}$ remained unreacted, even after $12 \mathrm{~h}$ at $90{ }^{\circ} \mathrm{C}$ and the intermediates monobromobisphenol A 8 , dibromobisphenol A 9 and tribromobisphenol A 10 were detected (Scheme 2 and Table 2). Interestingly, this partial reductive debromination occurred even under base-free conditions, where again the mono-, di- and tribromo derivatives were isolated as confirmed by the ${ }^{1} \mathrm{H}-\mathrm{NMR}$ and GC-MS spectra of the compounds.

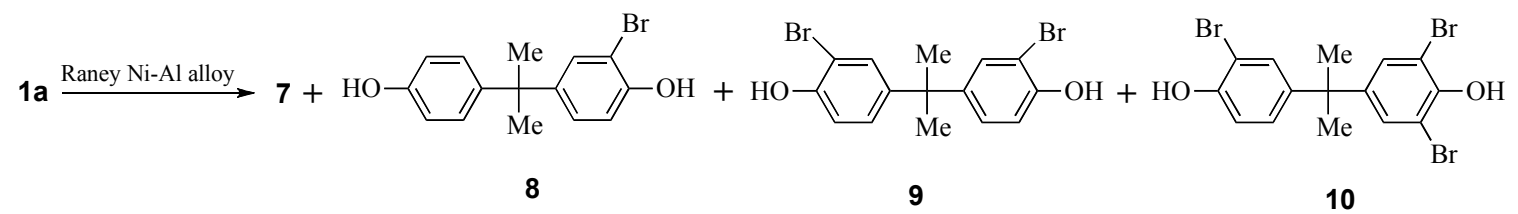

Scheme 2. Incomplete debromination - effect of base, amount of Raney Ni-Al alloy and temperature. 
Table 2. Effects of the concentration of alkaline solution, the amount of Raney Ni-Al alloy used and the reaction temperature in hydrodebromination of tetrabromobisphenol $1 \mathbf{a}^{\mathrm{a}}$

\begin{tabular}{|c|c|c|c|c|c|c|c|c|c|}
\hline \multicolumn{5}{|c|}{ Ratio $(\%)^{\mathrm{c}, \mathrm{d}}$} & \multirow[t]{2}{*}{ (h) } & \multirow{2}{*}{$\begin{array}{c}\text { Temp. } \\
\left({ }^{\circ} \mathrm{C}\right)\end{array}$} & \multirow{2}{*}{$\begin{array}{l}\text { Alkaline solution } \\
(\mathrm{mL})\end{array}$} & \multirow{2}{*}{$\begin{array}{l}\mathrm{Ni} / \mathrm{Al} \\
(\mathrm{g})\end{array}$} & \multirow[t]{2}{*}{ Run } \\
\hline 10 & 9 & 8 & 7 & 1a & & & & & \\
\hline 0.7 & 0.9 & 0.9 & 52.7 & 46.1 & 12 & 60 & $0.2 \% \mathrm{NaOH}(100) / \mathrm{H}_{2} \mathrm{O}(100)$ & 2 & 1 \\
\hline 0.5 & 1.5 & 0.3 & 3.0 & 94.7 & 12 & 60 & $\mathrm{H}_{2} \mathrm{O}(100) / \mathrm{H}_{2} \mathrm{O}(100)$ & 2 & 2 \\
\hline 7.7 & 4.0 & 3.6 & 5.8 & 78.9 & 12 & 90 & $\mathrm{H}_{2} \mathrm{O}(100) / \mathrm{H}_{2} \mathrm{O}(100)$ & 2 & 3 \\
\hline 10.7 & 11.9 & 17.7 & 9.6 & 50.1 & 12 & 90 & $\mathrm{H}_{2} \mathrm{O}(100) / \mathrm{H}_{2} \mathrm{O}(100)$ & 5 & 4 \\
\hline$(7.7)$ & $(8.2)$ & $(11.5)$ & $(4.4)$ & $(48.5)$ & & & & & \\
\hline
\end{tabular}

${ }^{\mathrm{a}} \mathbf{1 a}(2.0 \mathrm{mmol}, 1.09 \mathrm{~g}) .{ }^{\mathrm{b}}$ added within $1.0 \mathrm{~h} .{ }^{\mathrm{c}} \mathrm{GC}$ ratio. ${ }^{\mathrm{d}}$ isolated yields in parentheses.

Effect of the nature of the alloy and the effect of using metal powders in the reductive dehalogenation

Above, it was stated that Raney $\mathrm{Ni}-\mathrm{Al}$ alloy in a $1 \mathrm{wt} \%$ aq. $\mathrm{NaOH}$ solution is a very strong reducing agent. With this information in hand, the behaviour of other alloys such as $\mathrm{Co}-\mathrm{Al}, \mathrm{Cu}-$ $\mathrm{Al}$, and $\mathrm{Fe}-\mathrm{Al}$ was examined in the reductive debromination of 1a (Table 3). The hydrodebromination reaction was found to be slow with $\mathrm{Co}-\mathrm{Al}$ and $\mathrm{Cu}-\mathrm{Al}$ alloys. In both cases, a complete debromination of 1a could not be realized (Table 3, Runs 2 and 3). With Fe-Al alloy, the reductive debromination hardly occurred at all. Using $\mathrm{Al}$ ( $2.5 \mathrm{~g}$ for $2 \mathrm{mmol}$ substrate) and $\mathrm{Ni}$ ( $2.5 \mathrm{~g}$ for $2 \mathrm{mmol}$ substrate) or a combination of $\mathrm{Al}(2.5 \mathrm{~g})$ and $\mathrm{Ni}(2.5 \mathrm{~g})$ for $2 \mathrm{mmol}$ of substrate, the reduction did not take place, and $1 \mathrm{a}$ remained unreacted. These results clearly show that of the above, only Ni-Al alloy is highly effective for the reduction of $\mathbf{1 a}$.

Table 3. Effects of the alloys and metals in hydrodebromination of tetrabromobisphenol $1 \mathbf{a}^{\mathrm{a}}$

\begin{tabular}{ccccccccccccc}
\hline \multicolumn{10}{c}{ Ratio (\%) } & \multicolumn{1}{c}{ Time } & $\begin{array}{c}\text { Alloy } \\
(\mathrm{h})\end{array}$ & Run \\
\hline $\mathbf{1 0}$ & $\mathbf{9}$ & $\mathbf{8}$ & $\mathbf{7}$ & $\mathbf{6}$ & $\mathbf{5}$ & $\mathbf{4}$ & $\mathbf{3}$ & $\mathbf{2}$ & $\mathbf{1 a}$ & \\
\hline 0 & 0 & 0 & 55.0 & 6.0 & 23.4 & 0 & 8.1 & 7.5 & 0 & 6 & $\begin{array}{c}\mathrm{Ni} / \mathrm{Al} \\
(5)\end{array}$ & 1 \\
1.2 & 0.6 & 0.8 & 6.7 & 0 & 0 & 0 & 0 & 0 & 90.7 & 12 & $\begin{array}{c}\mathrm{Cu} / \mathrm{Al} \\
(5)\end{array}$ & 2 \\
0.6 & 0.7 & 0.2 & 4.5 & 0 & 0 & 0 & 0 & 0 & 93.9 & 12 & $\begin{array}{c}\mathrm{Co} / \mathrm{Al} \\
(5)\end{array}$ & 3 \\
0.4 & 0.3 & 0 & 0 & 0 & 0 & 0 & 0 & 0 & 98.3 & 12 & $\begin{array}{c}\mathrm{Fe} / \mathrm{Al} \\
(5)\end{array}$ & 4 \\
\hline
\end{tabular}

${ }^{\mathrm{a}} 1 \% \mathrm{NaOH}$ aq. solution $(100 \mathrm{~mL})$ was added to $1 \mathrm{a}(2.0 \mathrm{mmol}, 1.09 \mathrm{~g})$ in water $(100 \mathrm{~mL})$ at 90 ${ }^{\circ} \mathrm{C}$ within $1 \mathrm{~h} .{ }^{\mathrm{b}} \mathrm{GC}$ ratio. 
Dechlorination of tetrachlorobisphenol A 1b. Raney Ni-Al alloy is also effective in the reduction of tetrachlorobisphenol A $\mathbf{1 b}$ (Table 4). Here, a mixture of compounds 2, 3, 4, 5, 6, and 7 was afforded upon dropwise addition of a $1 \mathrm{wt} \%$ aqueous alkaline solution of $\mathrm{NaOH}, \mathrm{KOH}$, $\mathrm{CsOH}$ or $\mathrm{Ca}(\mathrm{OH})_{2}$ (Table 4, Runs 1-4). Bisphenol 7 was formed as the main product with small amounts of a mixture of compounds $\mathbf{2}, \mathbf{3}, \mathbf{4}, \mathbf{5}$, and $\mathbf{6}$, when a $0.2 \mathrm{wt} \%$ aq. solution of $\mathrm{NaOH}$, $\mathrm{KOH}, \mathrm{CsOH}$ or $\mathrm{Ca}(\mathrm{OH})_{2}$ was added at $90{ }^{\circ} \mathrm{C}$. These results are comparable to those of the debromination experiments mentioned above. Under ultrasonication at $60{ }^{\circ} \mathrm{C}$, $\mathbf{1 b}$ was dechlorinated both upon dropwise addition of a $1 \mathrm{wt} \%$ or a $0.2 \mathrm{wt} \%$ aq. $\mathrm{NaOH}$ solution.

Table 4. Hydrodechlorination of tetrachlorobisphenol $\mathbf{1 b}^{\mathrm{a}}$

\begin{tabular}{|c|c|c|c|c|c|c|c|c|c|c|}
\hline \multicolumn{6}{|c|}{ Ratio $(\%)^{\mathrm{c}, \mathrm{d}}$} & \multirow[t]{2}{*}{ (h) } & \multirow{2}{*}{$\begin{array}{l}\text { Temp. } \\
\left({ }^{\circ} \mathrm{C}\right)\end{array}$} & \multirow{2}{*}{$\begin{array}{l}\text { Alkaline solution }{ }^{\mathrm{b}} \\
(\mathrm{mL})\end{array}$} & \multirow{2}{*}{$\begin{array}{c}\mathrm{Ni} / \mathrm{Al} \\
(\mathrm{g})\end{array}$} & \multirow[t]{2}{*}{ Run } \\
\hline 7 & 6 & 5 & 4 & 3 & 2 & & & & & \\
\hline $\begin{array}{c}57.7 \\
(50.6)\end{array}$ & $\begin{array}{c}5.1 \\
(3.6)\end{array}$ & $\begin{array}{c}22.8 \\
(17.8)\end{array}$ & 0 & $7.3(4.6)$ & $7.1(5.0)$ & 6 & 90 & $\begin{array}{c}1 \% \mathrm{NaOH}(100) \\
/ \mathrm{H}_{2} \mathrm{O}(100)\end{array}$ & 5 & 1 \\
\hline 54.1 & 6.5 & 24.7 & 0 & 7.7 & 7.0 & 6 & 90 & $\begin{array}{c}1 \% \mathrm{KOH}(100) \\
/ \mathrm{H}_{2} \mathrm{O}(100)\end{array}$ & 5 & 2 \\
\hline 53.6 & 7.0 & 22.3 & 0 & 8.3 & 8.8 & 6 & 90 & $\begin{array}{c}1 \% \mathrm{CsOH}(100) \\
/ \mathrm{H}_{2} \mathrm{O}(100)\end{array}$ & 5 & 3 \\
\hline 60.9 & 5.1 & 21.1 & 0 & 7.9 & 5.0 & 7 & 90 & $\begin{array}{c}1 \% \mathrm{Ca}(\mathrm{OH})_{2}(100) \\
\quad / \mathrm{H}_{2} \mathrm{O}(100)\end{array}$ & 5 & 4 \\
\hline 91.2 & 7.0 & 0.9 & 0 & 0.4 & 0.5 & 5 & 90 & $\begin{array}{c}0.2 \% \mathrm{KOH}(100) \\
/ \mathrm{H}_{2} \mathrm{O}(100)\end{array}$ & 2 & 5 \\
\hline 91.7 & 6.3 & 1.3 & 0 & 0.4 & 0.3 & 5 & 90 & $\begin{array}{c}0.2 \% \mathrm{NaOH}(100) \\
/ \mathrm{H}_{2} \mathrm{O}(100)\end{array}$ & 2 & 6 \\
\hline 88.9 & 7.6 & 2.1 & 0 & 0.5 & 0.9 & 5 & 90 & $\begin{array}{c}0.2 \% \mathrm{CsOH}(100) \\
/ \mathrm{H}_{2} \mathrm{O}(100)\end{array}$ & 2 & 7 \\
\hline 93.5 & 3.2 & 2.0 & 0.4 & 0.3 & 0.6 & 6 & 90 & $\begin{array}{c}0.2 \% \mathrm{Ca}(\mathrm{OH})_{2}(100) \\
/ \mathrm{H}_{2} \mathrm{O}(100)\end{array}$ & 2 & 8 \\
\hline 75.0 & 3.0 & 7.5 & 2.0 & 1.5 & 1.2 & 7 & $60^{\mathrm{e}}$ & $\begin{array}{c}1 \% \mathrm{NaOH}(100) \\
/ \mathrm{H}_{2} \mathrm{O}(100)\end{array}$ & 5 & 9 \\
\hline $\begin{array}{c}100 \\
(94.6)\end{array}$ & 0 & 0 & 0 & 0 & 0 & 6 & $60^{\mathrm{e}}$ & $\begin{array}{c}0.2 \% \mathrm{NaOH}(100) \\
/ \mathrm{H}_{2} \mathrm{O}(100)\end{array}$ & 2 & 10 \\
\hline
\end{tabular}

${ }^{\mathrm{a}}$ 1a $(2.0 \mathrm{mmol}, 0.73 \mathrm{~g})$. ${ }^{\mathrm{b}}$ added within $1.0 \mathrm{~h} .{ }^{\mathrm{c}} \mathrm{GC}$ ratio. ${ }^{\mathrm{d}}$ isolated yields in parentheses. eultrasonication.

Dehalogenation of tetrabromo- and tetrachlorobisphenol A 1a,b with Raney Ni-Al Alloy in dilute aqueous solutions of alkali metal carbonates

Effects of the nature, the concentration and the amount of the aqueous alkali metal carbonate solutions. Based on the findings described above, we decided to investigate the 
behavior of the Raney Ni-Al alloy in much more weakly basic solutions such as in dilute aqueous solutions of alkali metal carbonates (Table 5). As in our observations for dilute aqueous alkaline solutions, the reducing capability of the Raney $\mathrm{Ni}-\mathrm{Al}$ alloy was enhanced greatly in dilute aqueous $\mathrm{Na}_{2} \mathrm{CO}_{3}$ solutions. In the case of using a 1 wt $\%$ aq. $\mathrm{Na}_{2} \mathrm{CO}_{3}$ solution, $1 \mathrm{a}$ was hydrodebrominated successfully to give a mixture of compounds $\mathbf{2}, \mathbf{3}, \mathbf{4 , 5}, \mathbf{6}$, and $\mathbf{7}$. Other alkali carbonates such as $\mathrm{K}_{2} \mathrm{CO}_{3}, \mathrm{Cs}_{2} \mathrm{CO}_{3}$ and $\mathrm{Li}_{2} \mathrm{CO}_{3}$ were also tested and the reductive debromination could be carried out successfully to produce a mixture of compounds $2,3,4,5,6$, and 7 .

Table 5. Effects of the nature and concentration of alkali metal carbonate solution for the dehalogenation of $\mathbf{1 a}, \mathbf{b}^{\text {a }}$

\begin{tabular}{|c|c|c|c|c|c|c|c|c|c|c|}
\hline \multicolumn{6}{|c|}{ Ratio $(\%)^{\mathrm{c}, \mathrm{d}}$} & \multirow[t]{2}{*}{ (h) } & \multirow{2}{*}{$\begin{array}{l}\text { Temp. } \\
\left({ }^{\circ} \mathrm{C}\right)\end{array}$} & \multirow{2}{*}{$\begin{array}{c}\mathrm{M}_{2} \mathrm{CO}_{3} \text { solution } \\
(\mathrm{mL})\end{array}$} & \multirow{2}{*}{$\begin{array}{c}\mathrm{Ni} / \mathrm{Al} \\
(\mathrm{g})\end{array}$} & \multirow{2}{*}{$\begin{array}{l}\text { Run } \\
\text { (Sub) }\end{array}$} \\
\hline 7 & 6 & 5 & 4 & 3 & 2 & & & & & \\
\hline 85.7 & 0.5 & 7.8 & 1.6 & 2.3 & 2.1 & 9 & 90 & $5 \% \mathrm{Na}_{2} \mathrm{CO}_{3}(100) / \mathrm{H}_{2} \mathrm{O}(100)$ & 5 & $\begin{array}{c}1 \\
(\mathbf{1 a})\end{array}$ \\
\hline $\begin{array}{c}68.6 \\
(59.8)\end{array}$ & 1.5 & $\begin{array}{c}16.7 \\
(11.1)\end{array}$ & 2.5 & $\begin{array}{c}6.0 \\
(3.2)\end{array}$ & $\begin{array}{c}6.2 \\
(3.6)\end{array}$ & 7 & 90 & $1 \% \mathrm{Na}_{2} \mathrm{CO}_{3}(100) / \mathrm{H}_{2} \mathrm{O}(100)$ & 5 & $\begin{array}{c}2 \\
(\mathbf{1 a})\end{array}$ \\
\hline 64.7 & 1.0 & 19.3 & 1.0 & 7.1 & 6.9 & 6 & 90 & $1 \% \mathrm{~K}_{2} \mathrm{CO}_{3}(100) / \mathrm{H}_{2} \mathrm{O}(100)$ & 5 & $\begin{array}{c}3 \\
(\mathbf{1 a})\end{array}$ \\
\hline 64.9 & 1.1 & 21.5 & 0.8 & 6.3 & 5.4 & 7 & 90 & $1 \% \mathrm{CsCO}_{3}(100) / \mathrm{H}_{2} \mathrm{O}(100)$ & 5 & $\begin{array}{c}4 \\
(\mathbf{1 a})\end{array}$ \\
\hline 86.6 & 0 & 11.2 & 1.1 & 0.7 & 0.4 & 8 & 90 & $1 \% \mathrm{Li}_{2} \mathrm{CO}_{3}(100) / \mathrm{H}_{2} \mathrm{O}(100)$ & 5 & $\begin{array}{c}5 \\
(\mathbf{1 a})\end{array}$ \\
\hline 82.6 & 1.3 & 15.6 & 1.0 & 0.3 & 0.2 & 8 & 90 & $1 \% \mathrm{Na}_{2} \mathrm{CO}_{3}(100) / \mathrm{H}_{2} \mathrm{O}(100)$ & 5 & $\begin{array}{c}6 \\
(\mathbf{1 b})\end{array}$ \\
\hline 78.2 & 1.6 & 18.1 & 0 & 0.9 & 1.2 & 8 & 90 & $1 \% \mathrm{~K}_{2} \mathrm{CO}_{3}(100) / \mathrm{H}_{2} \mathrm{O}(100)$ & 5 & $\begin{array}{c}7 \\
(\mathbf{1 b})\end{array}$ \\
\hline 76.5 & 1.9 & 19.3 & 0.4 & 0.3 & 1.6 & 8 & 90 & $1 \% \mathrm{CsCO}_{3}(100) / \mathrm{H}_{2} \mathrm{O}(100)$ & 5 & $\begin{array}{c}8 \\
(\mathbf{1 b})\end{array}$ \\
\hline 89.1 & 0 & 9.5 & 1.1 & 0.1 & 0.2 & 9 & 90 & $1 \% \mathrm{Li}_{2} \mathrm{CO}_{3}(100) / \mathrm{H}_{2} \mathrm{O}(100)$ & 5 & $\begin{array}{c}9 \\
(\mathbf{1 b})\end{array}$ \\
\hline
\end{tabular}

${ }^{\mathrm{a}} \mathbf{1 a}, \mathbf{1 b}(2.0 \mathrm{mmol}) .{ }^{\mathrm{b}}$ added within $1.0 \mathrm{~h} .{ }^{\mathrm{c}} \mathrm{GC}$ ratio. ${ }^{\mathrm{d}}$ isolated yields in parentheses.

\section{Debromination of 1a in other basic salt solutions}

The fact that Raney Ni-Al alloy is a very strong reducing agent in dilute aq. solutions of alkali metal carbonates (see above) encouraged us to investigate its reactivity in other aq. salt solutions (Table 6). In $1 \mathrm{wt} \%$ aq. solutions of $\left(\mathrm{NH}_{4}\right)_{2} \mathrm{CO}_{3}, \mathrm{NaHCO}_{3}$, and $\mathrm{KHCO}_{3}$, the reductive hydrodebromination of 1a was found to proceed very slowly (Table 6, Runs 1-3). 1a was partially debrominated to a mixture of mono-, di- and tribromide, and bisphenol A along with unchanged 1a. In $1 \mathrm{wt} \%$ aq. solutions of $\mathrm{NaOAc}, \mathrm{PhCO}_{2} \mathrm{Na}$, sodium oxalate and $\mathrm{NH}_{4} \mathrm{Cl}$, very 
little reductive hydrodebromination occurred. These results clearly show that Raney $\mathrm{Ni}-\mathrm{Al}$ alloy is a much stronger reducing agent in dilute aq. solutions of $\mathrm{NaOH}, \mathrm{KOH}, \mathrm{Na}_{2} \mathrm{CO}_{3}$ and $\mathrm{K}_{2} \mathrm{CO}_{3}$ than in other aq. basic salt solutions.

Table 6. Hydrodebromination of tetrabromobisphenol $1 \mathrm{a}^{\mathrm{a}}$

\begin{tabular}{|c|c|c|c|c|c|c|c|c|c|}
\hline \multicolumn{5}{|c|}{ Ratio $(\%)^{\mathrm{c}}$} & \multirow[t]{2}{*}{ (h) } & \multirow{2}{*}{$\begin{array}{c}\text { Temp. } \\
\left({ }^{\circ} \mathrm{C}\right)\end{array}$} & \multirow{2}{*}{$\begin{array}{l}\text { Salt solution } \\
\quad(\mathrm{mL})\end{array}$} & \multirow{2}{*}{$\begin{array}{c}\mathrm{Ni} / \mathrm{Al} \\
(\mathrm{g})\end{array}$} & \multirow[t]{2}{*}{ Run } \\
\hline 10 & 9 & 8 & 7 & $1 \mathrm{a}$ & & & & & \\
\hline 3.9 & 5.2 & 2.1 & 22.7 & 66.1 & 10 & 60 & $1 \%\left(\mathrm{NH}_{4}\right)_{2} \mathrm{CO}_{3}(100) / \mathrm{H}_{2} \mathrm{O}(100)$ & 5 & 1 \\
\hline 2.4 & 2.5 & 2.6 & 18.0 & 74.5 & 12 & 90 & $1 \% \mathrm{NaHCO}_{3}(100) / \mathrm{H}_{2} \mathrm{O}(100)$ & 5 & 2 \\
\hline 3.4 & 3.0 & 3.6 & 19.8 & 70.2 & 12 & 90 & $1 \% \mathrm{KHCO}_{3}(100) / \mathrm{H}_{2} \mathrm{O}(100)$ & 5 & 3 \\
\hline 5.7 & 0.9 & 0.7 & 6.6 & 86.1 & 12 & $60^{\mathrm{d}}$ & $1 \% \mathrm{KHCO}_{3}(100) / \mathrm{H}_{2} \mathrm{O}(100)$ & 5 & 4 \\
\hline 0.4 & 0.4 & 0 & 0 & 98.2 & 12 & 90 & $1 \% \mathrm{NaOAc}(100) / \mathrm{H}_{2} \mathrm{O}(100)$ & 5 & 5 \\
\hline 0.8 & 0.2 & 0 & 0 & 99.0 & 12 & 90 & $1 \% \mathrm{PhCO}_{2} \mathrm{Na}(100) / \mathrm{H}_{2} \mathrm{O}(100)$ & 5 & 6 \\
\hline 2.0 & 0.7 & 0 & 0 & 97.3 & 12 & 90 & $\begin{array}{c}\text { 1\%sodium oxalate } \\
(100) / \mathrm{H}_{2} \mathrm{O}(100)\end{array}$ & 5 & 7 \\
\hline 2.5 & 0.6 & 0 & 0 & 96.9 & 12 & 90 & $1 \% \mathrm{NH}_{4} \mathrm{Cl}(100) / \mathrm{H}_{2} \mathrm{O}(100)$ & 5 & 8 \\
\hline
\end{tabular}

${ }^{\mathrm{a}} \mathbf{1 a}(2.0 \mathrm{mmol}, 1.09 \mathrm{~g}){ }^{\mathrm{b}}$ added within $1.0 \mathrm{~h} .{ }^{\mathrm{c}} \mathrm{GC}$ ratio. ${ }^{\mathrm{d}}$ ultrasonication.

Hydrodebromination of polybromodiphenyl ethers with Raney Ni-Al alloy in dilute aqueous basic solutions

Debromination of polybromodiphenyl ethers with Raney Ni-Al alloy in dilute alkaline solutions

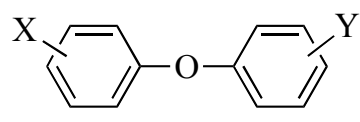

11a-h

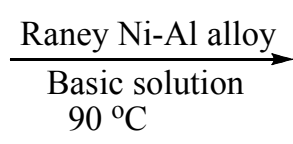
$90{ }^{\circ} \mathrm{C}$

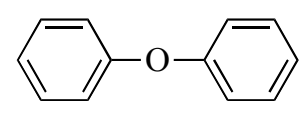

12

a: $\mathrm{X}=4-\mathrm{Br}, \mathrm{Y}=\mathrm{H} ; \mathbf{b}: \mathrm{X}=3-\mathrm{Br}, \mathrm{Y}=\mathrm{H} ; \mathbf{c}: \mathrm{X}=2-\mathrm{Br}, \mathrm{Y}=\mathrm{H} ; \mathbf{d}: \mathrm{X}=2,4-\mathrm{Br}_{2}, \mathrm{Y}=\mathrm{H}$;

$\mathbf{e}: \mathrm{X}=4-\mathrm{Br}, \mathrm{Y}=4^{\prime}-\mathrm{Br} ; \mathbf{f}: \mathrm{X}=3-\mathrm{Br}, \mathrm{Y}=3^{\prime}-\mathrm{Br} ; \mathbf{g}: \mathrm{X}=2,4-\mathrm{Br}_{2}, \mathrm{Y}=4^{\prime}-\mathrm{Br} ; \mathbf{h}: \mathrm{X}=2,4,6-\mathrm{Br}_{3}, \mathrm{Y}=\mathrm{H}$

Scheme 3. Reduction of halogenated diphenyl ethers with Raney Ni-Al alloy. 
Table 7. Hydrodebromination of polybromodiphenyl ethers 11a-h

\begin{tabular}{|c|c|c|c|c|c|c|}
\hline \multicolumn{2}{|c|}{ Yield $(\%)^{c}$} & \multirow[t]{2}{*}{ Time (h) } & \multirow[t]{2}{*}{ Basic solution $^{\mathrm{b}}(\mathrm{mL})$} & \multirow{2}{*}{$\begin{array}{c}\mathrm{Ni}-\mathrm{Al} \\
(\mathrm{g})\end{array}$} & \multirow[t]{2}{*}{ Sub. } & \multirow[t]{2}{*}{ Run } \\
\hline 12 & 11 & & & & & \\
\hline 80.8 & 19.2 & 8 & $1 \% \mathrm{NaOH}(100) / \mathrm{H}_{2} \mathrm{O}(100)$ & 0.5 & 11a & 1 \\
\hline $100(93.3)$ & 0 & 4 & $1 \% \mathrm{NaOH}(100) / \mathrm{H}_{2} \mathrm{O}(100)$ & 1.0 & $11 \mathrm{a}$ & 2 \\
\hline $100(92.1)$ & 0 & 4 & $1 \% \mathrm{KOH}(100) / \mathrm{H}_{2} \mathrm{O}(100)$ & 1.0 & 11a & 3 \\
\hline $100(92.4)$ & 0 & 4 & $1 \% \mathrm{CsOH}(100) / \mathrm{H}_{2} \mathrm{O}(100)$ & 1.0 & 11a & 4 \\
\hline $100(90.5)$ & 0 & 6 & $1 \% \mathrm{LiOH}(100) / \mathrm{H}_{2} \mathrm{O}(100)$ & 1.0 & $11 \mathrm{a}$ & 5 \\
\hline $100(92.1)$ & 0 & 5 & $1 \% \mathrm{Ca}(\mathrm{OH})_{2}(100) / \mathrm{H}_{2} \mathrm{O}(100)$ & 1.0 & $11 \mathrm{a}$ & 6 \\
\hline $100(91.5)$ & 0 & 5 & $0.2 \% \mathrm{NaOH}(100) / \mathrm{H}_{2} \mathrm{O}(100)$ & 0.5 & $11 \mathrm{a}$ & 7 \\
\hline $100(95.4)$ & 0 & 4 & $1 \% \mathrm{NaOH}(100) / \mathrm{H}_{2} \mathrm{O}(100)$ & 1.0 & $11 \mathrm{~b}$ & 8 \\
\hline $100(90.4)$ & 0 & 5 & $0.2 \% \mathrm{NaOH}(100) / \mathrm{H}_{2} \mathrm{O}(100)$ & 0.5 & $11 \mathrm{~b}$ & 9 \\
\hline $100(95.3)$ & 0 & 4 & $1 \% \mathrm{NaOH}(100) / \mathrm{H}_{2} \mathrm{O}(100)$ & 1.0 & $11 \mathrm{c}$ & 10 \\
\hline $100(93.1)$ & 0 & 5 & $0.2 \% \mathrm{NaOH}(100) / \mathrm{H}_{2} \mathrm{O}(100)$ & 0.5 & $11 \mathrm{c}$ & 11 \\
\hline $100(92.8)$ & 0 & 4 & $1 \% \mathrm{NaOH}(100) / \mathrm{H}_{2} \mathrm{O}(100)$ & 1.5 & 11d & 12 \\
\hline $100(93.8)$ & 0 & 6 & $0.2 \% \mathrm{NaOH}(100) / \mathrm{H}_{2} \mathrm{O}(100)$ & 1.0 & 11d & 13 \\
\hline $100(93.6)$ & 0 & 4 & $1 \% \mathrm{NaOH}(100) / \mathrm{H}_{2} \mathrm{O}(100)$ & 1.5 & $11 \mathrm{e}$ & 14 \\
\hline $100(94.6)$ & 0 & 6 & $0.2 \% \mathrm{NaOH}(100) / \mathrm{H}_{2} \mathrm{O}(100)$ & 1.0 & $11 \mathrm{e}$ & 15 \\
\hline $100(92.4)$ & 0 & 4 & $1 \% \mathrm{NaOH}(100) / \mathrm{H}_{2} \mathrm{O}(100)$ & 1.5 & $11 f$ & 16 \\
\hline $100(91.6)$ & 0 & 6 & $0.2 \% \mathrm{NaOH}(100) / \mathrm{H}_{2} \mathrm{O}(100)$ & 1.0 & $11 \mathrm{f}$ & 17 \\
\hline $100(93.2)$ & 0 & 5 & $1 \% \mathrm{NaOH}(100) / \mathrm{H}_{2} \mathrm{O}(100)$ & 2.0 & $11 \mathrm{~g}$ & 18 \\
\hline $100(92.1)$ & 0 & 7 & $0.2 \% \mathrm{NaOH}(100) / \mathrm{H}_{2} \mathrm{O}(100)$ & 1.0 & $11 \mathrm{~g}$ & 19 \\
\hline $100(91.5)$ & 0 & 5 & $1 \% \mathrm{NaOH}(100) / \mathrm{H}_{2} \mathrm{O}(100)$ & 2.0 & $11 \mathrm{~h}$ & 20 \\
\hline $100(93.6)$ & 0 & 8 & $0.2 \% \mathrm{NaOH}(100) / \mathrm{H}_{2} \mathrm{O}(100)$ & 1.0 & $11 \mathrm{~h}$ & 21 \\
\hline
\end{tabular}

${ }^{\mathrm{a}} \mathbf{1 1}(1.0 \mathrm{mmol}){ }^{\mathrm{b}}$ added dropwise within $1.0 \mathrm{~h}$. ${ }^{\mathrm{c}}$ isolated yield.

Our success in the dehalogenation of tetrabromo- and tetrachlorobisphenol A prompted us to test this powerful reducing system in the reductive debromination of polybromodiphenyl ethers 11a-h (Table 7). 4-Bromodiphenyl ether 11a was selected as a model substrate, and various reaction conditions were examined. First, upon dropwise addition of $1 \mathrm{wt} \%$ aq. $\mathrm{NaOH}, \mathrm{KOH}$, $\mathrm{CsOH}, \mathrm{LiOH}$ and $\mathrm{Ca}(\mathrm{OH})_{2}$ solutions, 11a was reductively debrominated to give the corresponding diphenyl ether 12 in high isolated yield (Table 7, Runs 2-6). When the amount of Raney Ni-Al alloy was decreased ( $0.5 \mathrm{~g} / 1 \mathrm{mmol}$ substrate), the debromination became sluggish and $19.2 \%$ of $11 \mathrm{a}$ remained unreacted even after $8 \mathrm{~h}$ (Table 7, Run 1 vs Run 2). However, in the case of dropwise addition of a more dilute, $0.2 \mathrm{wt} \%$ aq. $\mathrm{NaOH}$ solution, the complete hydrodebromination of 11a could be accomplished by employing the same amount of Raney NiAl alloy (0.5 g/1 mmol substrate) (Table 7, Run 7). Similary, 3- and 4-bromodiphenyl ethers 11b,c, dibromodiphenyl ethers 11d-f and tribromodiphenyl ethers $11 \mathrm{~g}, \mathbf{h}$ were reduced successfully to offer compound $\mathbf{1 2}$ as the sole product (Table 7, Run 8-21). 


\section{Debromination of polybromodiphenyl ethers with Raney Ni-Al Alloy in dilute aqueous} solutions of alkali metal carbonates

Table 7. Hydrodebromination of polybromodiphenyl ethers 11a-h in solution of alkali metal carbonates $^{\mathrm{a}}$

\begin{tabular}{|c|c|c|c|c|c|c|}
\hline \multicolumn{2}{|c|}{ Yield $(\%)^{\mathrm{c}}$} & \multirow{2}{*}{$\begin{array}{l}\text { Time } \\
\text { (h) }\end{array}$} & \multirow{2}{*}{$\begin{array}{c}\text { Basic solution }^{\mathrm{b}} \\
(\mathrm{mL})\end{array}$} & \multirow{2}{*}{$\begin{array}{c}\mathrm{Ni}-\mathrm{Al} \\
(\mathrm{g})\end{array}$} & \multirow[t]{2}{*}{ Sub. } & \multirow[t]{2}{*}{ Run } \\
\hline 12 & 11 & & & & & \\
\hline 76.8 & 23.2 & 8 & $1 \% \mathrm{~K}_{2} \mathrm{CO}_{3}(100) / \mathrm{H}_{2} \mathrm{O}(100)$ & 0.5 & $11 \mathrm{a}$ & 1 \\
\hline $100(94.1)$ & 0 & 5 & $1 \% \mathrm{~K}_{2} \mathrm{CO}_{3}(100) / \mathrm{H}_{2} \mathrm{O}(100)$ & 1.0 & $11 \mathrm{a}$ & 2 \\
\hline $100(93.4)$ & 0 & 5 & $1 \% \mathrm{Na}_{2} \mathrm{CO}_{3}(100) / \mathrm{H}_{2} \mathrm{O}(100)$ & 1.0 & $11 \mathrm{a}$ & 3 \\
\hline $100(93.5)$ & 0 & 5 & $1 \% \mathrm{Cs}_{2} \mathrm{CO}_{3}(100) / \mathrm{H}_{2} \mathrm{O}(100)$ & 1.0 & $11 \mathrm{a}$ & 4 \\
\hline $100(92.1)$ & 0 & 6 & $1 \% \mathrm{Li}_{2} \mathrm{CO}_{3}(100) / \mathrm{H}_{2} \mathrm{O}(100)$ & 1.0 & $11 \mathrm{a}$ & 5 \\
\hline $100(94.2)$ & 0 & 5 & $0.2 \% \mathrm{Na}_{2} \mathrm{CO}_{3}(100) / \mathrm{H}_{2} \mathrm{O}(100)$ & 0.5 & $11 \mathrm{a}$ & 6 \\
\hline $100(95.4)$ & 0 & 5 & $1 \% \mathrm{Na}_{2} \mathrm{CO}_{3}(100) / \mathrm{H}_{2} \mathrm{O}(100)$ & 1.0 & $11 b$ & 7 \\
\hline $100(90.4)$ & 0 & 6 & $0.2 \% \mathrm{Na}_{2} \mathrm{CO}_{3}(100) / \mathrm{H}_{2} \mathrm{O}(100)$ & 0.5 & $11 b$ & 8 \\
\hline $100(95.3)$ & 0 & 5 & $1 \% \mathrm{Na}_{2} \mathrm{CO}_{3}(100) / \mathrm{H}_{2} \mathrm{O}(100)$ & 1.0 & $11 \mathrm{c}$ & 9 \\
\hline $100(93.1)$ & 0 & 5 & $1 \% \mathrm{Na}_{2} \mathrm{CO}_{3}(100) / \mathrm{H}_{2} \mathrm{O}(100)$ & 0.5 & $11 \mathrm{c}$ & 10 \\
\hline $100(92.8)$ & 0 & 5 & $1 \% \mathrm{Na}_{2} \mathrm{CO}_{3}(100) / \mathrm{H}_{2} \mathrm{O}(100)$ & 1.5 & 11d & 11 \\
\hline $100(94.6)$ & 0 & 6 & $1 \% \mathrm{Na}_{2} \mathrm{CO}_{3}(100) / \mathrm{H}_{2} \mathrm{O}(100)$ & 1.5 & $11 \mathrm{e}$ & 12 \\
\hline $100(95.6)$ & 0 & 8 & $1 \% \mathrm{Na}_{2} \mathrm{CO}_{3}(100) / \mathrm{H}_{2} \mathrm{O}(100)$ & 1.5 & $11 f$ & 13 \\
\hline $100(93.2)$ & 0 & 6 & $1 \% \mathrm{Na}_{2} \mathrm{CO}_{3}(100) / \mathrm{H}_{2} \mathrm{O}(100)$ & 2.0 & $11 \mathrm{~g}$ & 14 \\
\hline $100(92.6)$ & 0 & 8 & $0.2 \% \mathrm{Na}_{2} \mathrm{CO}_{3}(100) / \mathrm{H}_{2} \mathrm{O}(100)$ & 1.0 & $11 \mathrm{~g}$ & 15 \\
\hline $100(92.5)$ & 0 & 8 & $1 \% \mathrm{NaOH}(100) / \mathrm{H}_{2} \mathrm{O}(100)$ & 2.0 & $11 \mathrm{~h}$ & 16 \\
\hline $100(91.6)$ & 0 & 9 & $0.2 \% \mathrm{Na}_{2} \mathrm{CO}_{3}(100) / \mathrm{H}_{2} \mathrm{O}(100)$ & 1.0 & $11 \mathrm{~h}$ & 17 \\
\hline
\end{tabular}

${ }^{\mathrm{a}} \mathbf{1 1}(1.0 \mathrm{mmol})$. ${ }^{\mathrm{b}}$ added dropwise within $1.0 \mathrm{~h}$. ${ }^{\mathrm{c}}$ isolated yield.

The dehalogenation reaction was carried out by slowly adding an aqueous alkaline solution to a mixture of halogenated compounds 11a-h, Raney Ni-Al alloy and water at $90{ }^{\circ} \mathrm{C}$. As model reductions of polybromodiphenyl ethers, the reductive debrominations of monobromodiphenyl 11a-c, dibromodiphenyl ether 11d-f and tribromodiphenyl ethers 11g,h were investigated (Table 8). By using $0.5 \mathrm{~g}$ of Raney Ni-Al alloy, 4-bromodiphenyl ether 11a was reduced to diphenyl ether 12 upon dropwise addition of $1 \mathrm{wt} \%$ aq. $\mathrm{K}_{2} \mathrm{CO}_{3}$. However, some 11 a remained unreacted (GC ratio: 23.2 area\%), after the reaction mixture had been heated for $7 \mathrm{~h}$ (Table 8, Run 1). Even when the reaction time was prolonged to $12 \mathrm{~h}$, 11a still remained. However, an increase of Raney Ni-Al alloy (1.0 g) resulted in complete consumption of 11a (Table 8, Run 3). 11a was also hydrodebrominated easily to give diphenyl ether 12 selectively and in high yield upon addition of a $1 \mathrm{wt} \%$ aq. $\mathrm{Na}_{2} \mathrm{CO}_{3}, \mathrm{Cs}_{2} \mathrm{CO}_{3}$ or $\mathrm{Li}_{2} \mathrm{CO}_{3}$ solution (Table 8, Runs 4-6). Similary, 3and 4-bromodiphenyl ethers $\mathbf{1 1 b}, \mathbf{c}$, dibromodiphenyl ethers 11d-f and tribromodiphenyl ethers 
11g,h were reduced successfully to produce compound $\mathbf{1 2}$ as the sole product (Table 8, Runs 819).

\section{Reaction Mechanism}

Although the reaction mechanism of the dehalogenation is not totally clarified yet, it can be proposed that aluminum metal of the Raney Ni-Al alloy reacts with the basic solution, e.g., with the aq. $\mathrm{NaOH}$ solution, and produces a reactive form of hydrogen and highly porous nickel metal on the catalyst's surface. The actual hydrodehalogenation may well operate as a mixture of ionic and radical pathways as has been suggested for the hydrodechlorination of chloroarenes over another supported nickel catalyst. ${ }^{23}$ In the case of ionic pathways the basicity of the solvent would exert an effect that would overlie the effect of the base on the production of the catalytic surface (Figure 1).

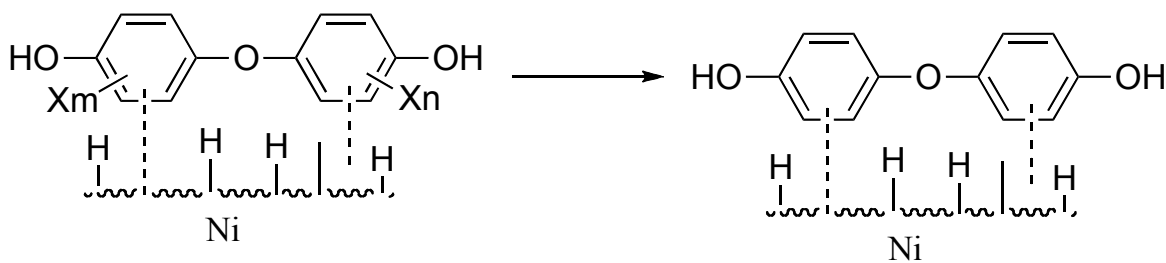

Figure 1. Schematic representation of the approach of the diphenyl ether to the metal surface.

Tetrachloro- and tetrabromobisphenol A, and polybromodiphenyl ethers, which are $p$ electron rich, are adsorbed on the surface of the active nickel metal, and reaction with the reactive form of hydrogen occurs at the catalyst's surface. We have noted that in dilute aq. $\mathrm{NaOH}, \mathrm{KOH}, \mathrm{Na}_{2} \mathrm{CO}_{3}$ and $\mathrm{K}_{2} \mathrm{CO}_{3}$ solutions, Raney Ni-Al alloy becomes a stronger reductant than in more concentrated alkaline solutions. The reasons for this may be a solution-dependent particle size distribution of active nickel metal, which plays an important role in the reductive dehalogenation reaction, and a different surface porosity of the catalyst, although both of these still need to be substantiated. In the case of using a $5 \mathrm{wt} \%$ aq. $\mathrm{Na}_{2} \mathrm{CO}_{3}$ solution, the Raney Ni-Al alloy reacts more uncontrolled with the $\mathrm{Na}_{2} \mathrm{CO}_{3}$ solution and gives off hydrogen gas, which escapes unreacted from the reaction vessel. In fact, an effervescence of hydrogen gas was observed in that case.

\section{Conclusions}

In conclusion, it was found that Raney $\mathrm{Ni}-\mathrm{Al}$ alloy in dilute solutions of alkali hydroxides and alkali metal carbonates becomes a powerful reducing agent. This is especially true in $0.5 \mathrm{wt} \%$ aq. $\mathrm{NaOH}, \mathrm{KOH}, \mathrm{Na}_{2} \mathrm{CO}_{3}$, and $\mathrm{K}_{2} \mathrm{CO}_{3}$ solutions. We have developed a new and efficient method for the dehydrohalogenation of PBDPEs, TBBPA and TCBPA, using commercially available Raney 
Ni-Al alloy. PBDPEs were dehydrobrominated to afford diphenyl ether 12. TBBPA and TCBPA afforded bisphenol A 7, together with smaller amounts of cyclohexanol 2, 4isopropylcyclohexanol 3, phenol 4, 4-isopropylphenol 5, and 2-hydroxyphenyl-2-phenylpropane 6. The ratio of the dehalogenation products was found to depend strongly on the amount of the Raney Ni-Al alloy used. The advantages of the present approach lie in the ease of manipulation, rapidity of the reaction, and the mildness of reaction conditions. The mild conditions used for the degradation of PBDPEs, TBBPA and TCBPA as compared to existing methods, especially in regard to temperature and reaction medium, should make this low cost protocol a valuable alternative. We believe that Raney Ni-Al alloy in dilute aqueous alkaline and alkali metal carbonate solutions provides another very useful reagent for the reductive dehalogenation of aromatic halides and for the hydrogenation of aromatic compounds. Raney Ni-Al alloy is readily available commercially and is, of course, cheaper than the Raney Ni catalyst made from it. It is expected that this procedure would have potential application for the disposal of toxic polyhalogenated arenes in certain cases in the industry. Further work on the reductive degradation of polychlorinated and brominated compounds is currently underway in our laboratory.

\section{Experimental Section}

General. ${ }^{1} \mathrm{H}$ NMR spectra were recorded with a JEOL EX-270 spectrometer $\left({ }^{1} \mathrm{H}\right.$ at $\left.270 \mathrm{MHz}\right)$. The chemical shifts are relative to TMS (solvent $\mathrm{CDCl}_{3}$, unless otherwise noted). Mass spectra were measured with a JMS-01-SG-2 spectrometer [electron impact mode (EI), $70 \mathrm{eV}$ or fast atom bombardment (FAB)] and with a GC-MS 6890[GC]/HP MS5973 combination. The GCdata given in Tables 1-8 are GC ratios of compounds $\mathbf{1}-\mathbf{1 2}$ and do not encompass minor sideproducts that may have been formed (normated to $100 \%$ ). To validate the GC data of the reaction mixture in the actual organic solvent extract as matrix, actual substance isolations of compounds 1 - 12 have been performed. For the two main components of interest, bisphenol-A and TBBPA, the accuracy can be given as $+/-0.07$ GC-ratio $\%$ for TBBPA and TCBPA 1a,b and $+/-0.04 \mathrm{GC}-$ ratio\% for BPA 7 within the range of concentrations obtained in this study. The reductive dehalogenation was monitored by a Shimadzu GC-17A gas chromatograph (Column: J \& W Scientific DB-1, $30 \mathrm{~m} \times 0.25 \mathrm{~mm} \times 0.25 \mathrm{um}$ ), equipped with a capillary column (stationary phase: dimethylpolysiloxane), using a hydrogen flame ionization detector. The relative ratio was calculated on the basis of the peak area of the GC (assuming equal FID-sensitivity for all substrates).

Raney Ni-Al, Co-Al, Cu-Al and Fe-Al (50:50, wt\%, particle size 80-100 mesh) $\mathrm{Al}$ and $\mathrm{Ni}$ powder (particle size 80-100 mesh) were bought from Wako Chemical Industry, Ltd., Japan. TBBPA 1a and TCBPA 1b were purchased from Haihua Risheng Chemical Co., Ltd. Suzhou, China. Bisphenol A 7, diphenyl ether 12, 4-bromodiphenyl ether 11c and 4,4'-dibromodiphenyl ether 11e (purchased from Aldrich) and 2-(3-bromo-4-hydroxyphenyl)-2-(3,5-dibromo-4- 
hydroxylphenyl)propane $\mathbf{1 0}^{24}$ (prepared by bromination of bisphenol A 7 with bromine in acetic acid as reported) were used as authentic samples. 2-(3-Bromo-4-hydroxyphenyl)-2-(4-hydroxylphenyl)propane $\quad \mathbf{8}^{25}, \quad$ 2,2-bis(3-bromo-4-hydroxyphenyl)propane $\quad \mathbf{9}^{25}$ and 2-(3-bromo-4hydroxyphenyl)-2-(3,5-dibromo-4-hydroxylphenyl)propane 10 were isolated and their structures were assigned on the basis of their ${ }^{1} \mathrm{H}$ NMR and GC-MS spectroscopic data. 2-Bromodiphenyl ether 11c, 3-bromodiphenyl ether 11b and 2,4,6-tribromodiphenyl ether $\mathbf{1 1 h}$ were prepared as reported in the literature. ${ }^{26}$

\section{Debromination of tetrabromobisphenol A 1a. Typical procedure}

An aq. $1 \mathrm{wt} \% \mathrm{~K}_{2} \mathrm{CO}_{3}$ solution $(50 \mathrm{ml})$ was added to a mixture of $1 \mathbf{a}$ (2.0 mmol, $\left.1.09 \mathrm{~g}\right)$, Raney $\mathrm{Ni}$-Al alloy $(1.0 \mathrm{~g})$ and water $(50 \mathrm{ml})$ within 1.0 hour and at $90{ }^{\circ} \mathrm{C}$. After the mixture was heated for an additional $7 \mathrm{~h}$ at $90{ }^{\circ} \mathrm{C}$, it was cooled to room temperature and filtered through Celite. The filtrate was extracted with ethyl acetate $(3 \times 15 \mathrm{ml})$ and the organic layer was dried over anhydrous magnesium sulfate. After removal of the solvent, a mixture of 2, 3, 4, 5, 6, 7, 8, 9 and 10 was obtained in the ratio of 6.2:6.0:2.5:16.7:1.5:67.1 (GC area\%) (Table 5, Run 2). Compounds 2, 3, 4, 5, 6, 7, 8, 9 and 10 were separated by column chromatography on silica gel and their structures were assigned on the basis of IR, ${ }^{1} \mathrm{H}$ NMR and GC-MS spectroscopic data and also compared in their GC retention time with authentic samples.

2-(3-Bromo-4-hydroxyphenyl)-2-(4-hydroxylphenyl)-propane 8. $\delta_{\mathrm{H}}\left(270 \mathrm{MHz}, \mathrm{CDCl}_{3}\right) 1.56$ $\left(6 \mathrm{H}, \mathrm{s}, 2 \mathrm{CH}_{3}\right), 5.26(1 \mathrm{H}, \mathrm{br}, \mathrm{OH}), 5.46(1 \mathrm{H}, \mathrm{br}, \mathrm{OH}), 6.70(2 \mathrm{H}, \mathrm{dt}, J=2.6,8.6 \mathrm{~Hz}, \mathrm{Ar}-\mathrm{H}), 6.82$ $(1 \mathrm{H}, \mathrm{d}, J=8.2 \mathrm{~Hz}, \mathrm{Ar}-\mathrm{H}), 7.02(1 \mathrm{H}, \mathrm{dd}, J=2.2,8.2 \mathrm{~Hz}, \mathrm{Ar}-\mathrm{H}), 7.06(2 \mathrm{H}, \mathrm{dt}, J=2.6,8.6 \mathrm{~Hz}, \mathrm{Ar}-$ $\mathrm{H}), 7.16(1 \mathrm{H}, \mathrm{d}, J=2.2 \mathrm{~Hz})$; MS: $308\left(\left[{ }^{81} \mathrm{Br}\right] \mathrm{M}^{+}\right), 306\left(\left[{ }^{79} \mathrm{Br}\right] \mathrm{M}^{+}\right), 293\left(\left[{ }^{81} \mathrm{Br}\right] \mathrm{M}-\mathrm{CH}_{3}\right), 291$ $\left(\left[{ }^{79} \mathrm{Br}\right] \mathrm{M}-\mathrm{CH}_{3}\right), 212,152,131,112$.

2,2-Bis(3-bromo-4-hydroxyphenyl)propane 9. $\delta_{\mathrm{H}}\left(270 \mathrm{MHz}, \mathrm{CDCl}_{3}\right) 1.56\left(6 \mathrm{H}, \mathrm{s}, 2 \mathrm{CH}_{3}\right), 5.44$ $(2 \mathrm{H}, \mathrm{br}, \mathrm{OH}), 6.86(2 \mathrm{H}, \mathrm{d}, J=8.6 \mathrm{~Hz}, \mathrm{Ar}-\mathrm{H}), 7.04(2 \mathrm{H}, \mathrm{dd}, J=2.2,8.6 \mathrm{~Hz}, \mathrm{Ar}-\mathrm{H}), 7.18(2 \mathrm{H}, \mathrm{d}, J$ $=2.2 \mathrm{~Hz}, \mathrm{Ar}-\mathrm{H})$; MS: $388\left(\left[{ }^{81} \mathrm{Br}_{2}\right] \mathrm{M}^{+}\right), 386\left(\left[{ }^{81} \mathrm{Br}^{79} \mathrm{Br}^{+} \mathrm{M}^{+}\right), 384\left(\left[{ }^{79} \mathrm{Br}_{2}\right] \mathrm{M}^{+}\right), 373\left(\left[{ }^{81} \mathrm{Br}_{2}\right] \mathrm{M}^{+}-\right.\right.$ $\left.\mathrm{CH}_{3}\right), 371\left(\left[{ }^{79} \mathrm{Br}_{2}\right] \mathrm{M}^{+}-\mathrm{CH}_{3}\right), 369\left(\left[{ }^{81} \mathrm{Br}^{79} \mathrm{Br}\right] \mathrm{M}^{+}-\mathrm{CH}_{3}\right), 292,290,211,152,131,112$.

2-(3-Bromo-4-hydroxyphenyl)-2-(3,5-dibromo-4-hydroxyphenyl)propane $10 .{ }^{\mathbf{2 4}} \delta_{\mathrm{H}}(270 \mathrm{MHz}$, $\left.\mathrm{CDCl}_{3}\right) 1.58\left(6 \mathrm{H}, \mathrm{s}, 2 \mathrm{CH}_{3}\right), 5.42(1 \mathrm{H}, \mathrm{br}, \mathrm{OH}), 5.74(1 \mathrm{H}, \mathrm{br}, \mathrm{OH}), 6.90(1 \mathrm{H}, \mathrm{d}, J=8.6 \mathrm{~Hz}, \mathrm{Ar}-$ H), 7.00 (1H, dd, $J=2.2,8.6 \mathrm{~Hz}, \mathrm{Ar}-\mathrm{H}), 7.26(2 \mathrm{H}, \mathrm{s}, \mathrm{Ar}-\mathrm{H}), 7.32$ (1H, d, J=2.2, Ar-H); MS: 468 $\left(\left[{ }^{81} \mathrm{Br}_{3}\right] \mathrm{M}^{+}\right), 466\left(\left[{ }^{81} \mathrm{Br}_{2}{ }^{79} \mathrm{Br}^{+} \mathrm{M}^{+}\right), 464\left(\left[{ }^{81} \mathrm{Br}^{79} \mathrm{Br}_{2}\right] \mathrm{M}^{+}\right), 462\left(\left[{ }^{79} \mathrm{Br}_{3}\right] \mathrm{M}^{+}\right), 453\left(\left[{ }^{81} \mathrm{Br}_{3}\right] \mathrm{M}^{+}-\mathrm{CH}_{3}\right)\right.$, 451 $\left(\left[{ }^{81} \mathrm{Br}_{2}{ }^{79} \mathrm{Br}\right] \mathrm{M}^{+}-\mathrm{CH}_{3}\right), 449\left(\left[{ }^{81} \mathrm{Br}^{79} \mathrm{Br}_{2}\right] \mathrm{M}^{+}-\mathrm{CH}_{3}\right), 447\left(\left[{ }^{79} \mathrm{Br}_{3}\right] \mathrm{M}^{+}-\mathrm{CH}_{3}\right), 372,370,368,291$, $289,210,152,131,112$.

\section{References}

1. (a) Hornung, A.; Balabanovich, A. I.; Donner, S.; Seifert, H. J. Anal. Appl. Pyrolysis 2003, 70, 723 and ref. cited therein. (b) Rahm, S.; Green, N.; Norrgran, J.; Bergman, A. Environ. Sci. Technol. 2005, 39, 3128. (c) de Wit, C. A. Chemosphere 2002, 46, 583. 
2. (a) Law, R. J.; Allchin, C. R.; de Boer, J.; Covaci, A.; Herzke, D.; Lepom, P.; Morris, S.; Tronczynski, J.; de Wit, C. A. Chemosphere 2006, 64, 187. (b) Haglund, P S.; Zook, D. R.; Buser, H.-R.; Hu, J. Environ. Sci. Technol. 1997, 31, 3281.

3. (a) Karlsson, M.; Julander, A.; van Bavel, B.; Hardell, L. Environ. Inter. 2007, 33, 62. (b) Sjoedin, A.; Patterson, D. G.; Bergman, A. Environ. Sci. Technol. 2001, 35, 3830. (c) Fernandez, M. F.; Araque, P.; Kiviranta, H.; Molina-Molina, J. M.; Rantakokko, P.; Laine, O.; Vartiainen, T.; Olea, N. Chemosphere 2007, 66, 377 and ref. cited therein.

4. (a) Tada, Y.; Fujitami, T.; Ogata, A.; Kamimura, H.; Ogata, A.; Kamimura, K. Environ. Toxicol. Pharmacol. 2007, 23, 174. (b) Canton, R. E.; Sanderson, J. T.; Nijneijer, S.; Bergman, A.; Letcher, R. J.; van der Berg, M. Toxicol. Appl. Pharmacol. 2006, 216, 27.

5. Santillo, D.; Johnston, P. Environ. Internat. 2003, 29, 725.

6. (a) Balabanovich, A. I.; Hornung, A.; Luda, M. P.; Koch, W.; Tumiatti, V. Environ. Sci. Technol. 2005, 39, 5469. (b) Barontini, F.; Cozzani, V.; Marsanich, K.; Raffa, V.; Petarca, L. J. Anal. Appl. Pyrolysis 2004, 72, 41. (c) Marsanich, K.; Zanelli, S.; Barontini, F.; Cazzoni, V. Thermochim. Acta 2004, 421, 95.

7. (a) Lai, Y.-C.; Lee, W.-J.; Li, H-W.; Wang, L.-C.; Chang-Chien, G.-P. Environ. Sci. Technol. 2007, 41, 957. (b) Barontini, F.; Cozzani, V. J. Anal. Appl. Pyrolysis 2006, 77, 41. (c) Balabanovich, A. I.; Hornung, A.; Lunda, M. P.; Koch, W.; Tumiatti, V. Environ. Sci. Technol. 2005, 39, 5469. (d) Ebert, J.; Bahadir, M. Environ. Inter. 2003, 29, 711. (e) Söderström, G.; Marklund, S. Environ. Sci. Technol. 2002, 36, 1959.

8. Hornung, A.; Donner, S.; Balabanovich, A.; Seifert, H. J. Cleaner Prod. 2005, 13, 525.

9. Mackenzie, K.; Kopinke, F. D. Chemosphere 1996, 33, 2423.

10. Brodersen, K.; Tartler, D.; Danzer, B. Wertstoffrückgewinnung aus Vielstoffgemischen am Beispiel Computerschrott, In BayForrest, Bayrischer Forschungsverbund Abfallforschung und Reststoffverwertung, Berichtsheft 4, Wilderer, P. A.; Koralewska, R., Eds., 3. Statusseminar, München, 1995, p 227.

11. Adimurthy, S.; Vaghela, S. S.; Vyas, P. V.; Bhatt, A. K.; Ramachandraiah, G.; Bedekar, A. V. Tetrahedron Lett. 2003, 44, 6393.

12. (a) Ahn, M.-Y.; Filley, T. R.; Jafvert, C. T.; Nies, L.; Hua, I.; Bezares-Cruz, J. Environ. Sci. Technol. 2006, 40, 215. (b) Keum, Y.-S.; Li, Q. X. Environ. Sci. Technol. 2005, 39, 2280. (c) Bezares-Cruz, J.; Jafvert, C. T.; Hua, I. Environ. Sci. Technol. 2004, 38, 4149. (d) Eriksson, J.; Rahm, S.; Green, N.; Bergman, Å.; Jakobsson, E. Chemosphere 2004, 54, 117.

13. Sun, C.; Zhao, D.; Ma, W.; Zhao, J. Environ. Sci. Technol. 2009, 43, 157.

14. Brebu, M.; Sakata, Y. Green Chem. 2006, 8, 984.

15. (a) Zinovyev, S. S.; Shinkova, N. A.; Perosa, A.; Tundo, P. Appl. Cat. B: Environmental 2005, 55, 49. (b) Zinovyev, S. S.; Shinkova, N. A.; Perosa, A.; Tundo, P. Appl. Cat. B: Environmental 2005, 55, 39. (c) Zinovyev, S. S.; Shinkova, N. A.; Perosa, A.; Tundo, P. Appl. Cat. B: Environmental 2004, 47, 27. (d) Zinovyev, S. S.; Shinkova, N. A.; Perosa, A.; Tundo, P. Appl. Cat. A: General 2004, 271, 129. (e) Tundo, P.; Perosa, A. React. Func. 
Polym. 2003, 54, 95. (f) Keefer, L. K. Lunn, G. Chem. Rev. 1989, 89, 459 and references therein..

16. (a) Liu, G.-B.; Tashiro, M.; Thiemann, T. Tetrahedron 2009, 65, 2497. (b) Liu, G.-B.; Dai, L.; Gao, X.; Li, M.-K.; Thiemann, T. Green Chem. 2006, 8, 781. (c) Liu, G.-B.; Tsukinoki, T.; Kanda, T.; Mitoma, Y.; Tashiro, M. Tetrahedron Lett. 1998, 39, 5991.

17. Fukuda, T.; Uchida, H.; Suzuki, M.; Miyamoto, H.; Morinaga, H.; Nawata, H.; Uwajima, T. J. Chem. Technol. Biotechnol. 2004, 79, 1212.

18. Hunter, S. E.; Savage, P. E. J. Org. Chem. 2004, 69, 4724.

19. Watanabe, H.; Horikoshi, S.; Kawabe, H.; Sugie, Y.; Zhao, J. C.; Hidaka, H. Chemosphere 2003, 52, 851 .

20. (a) Hunter, S. E.; Savage, P. E. Chem. Eng. Sci. 2004, 59, 4903. (b) Schnell, H.; Krimm, H. Angew. Chem., Int. Ed. Engl. 1963, 2, 373.

21. Pan, Y.; Holmes, C. P. Org. Lett. 2001, 3, 2769.

22. Petrocelli, F. P.; Klein, M. T. Fuel Sci. Technol. 1987, 5, 25.

23. Yakovlev, V. A.; Terskikh, V. V.; Simagina, V. I.; Likholobov, V. A. J. Mol. Catal. A 2000, $153,231$.

24. Eriksson, J.; Rahm, S.; Green, N.; Bergman, A.; Jakobsson, E. Chemosphere 2004, 54, 117.

25. Owens, Jr., C. V.; Lambright, C.; Bobseine, K.; Ryan, B.; Gray Jr., L. E.; Gullett, B. K.; Wilson, V. S. Environ. Sci. Technol. 2007, 41, 8506.

26. Marsh, G.; Hu, J.; Jakobsson, E.; Rahm, S.; Bergman, A. Environ. Sci. Technol. 1999, 33, 3033. 